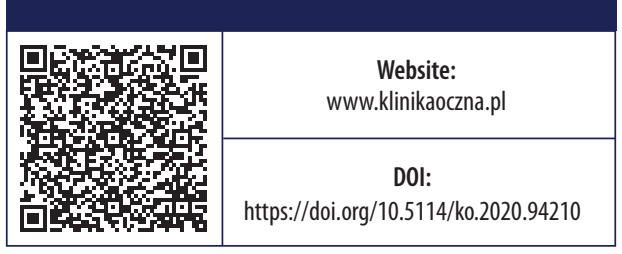

\title{
Ophthalmologic symptoms and diagnosis of a patient with Jansky-Bielschowsky disease
}

\author{
Monika Modrzejewska, Marta Kirkiewicz, Adriana Kiszkielis, Wojciech Lubiński \\ The $2^{\text {nd }}$ Department of Ophthalmology, the Pomeranian Medical University, Szczecin, Poland
}

\begin{abstract}
Introduction: Type 2 neuronal ceroid lipofuscinosis (CLN2) is a rare lysosomal neurodegenerative disease in which ceroid accumulating mainly in nerve cells. The disease manifests itself in the first years of a child's life as convulsions, epilepsy, gradual deterioration of motor function with gradual visual acuity impairment.

Case report: In a five-year-old patient with divergent squint, nystagmus, epilepsy confirmed in EEG and with significance decrease in visual acuity, ophthalmological examinations confirmed at irregular, re-arrangement of dye, covering the macular region indicating the presence of the bull's eye maculopathy. Additional changes were features of pallor of optic nerve disc with generalized retinal vessels vasoconstriction. In electrophysiological tests of fERG, damage of the bioelectrophysiological function of the retina indicating cone-rod like degeneration was recorded. In lab tests,
\end{abstract}

reduction of lysosomal enzyme activity - tripeptidyl peptidase (TPP1) in leukocytes (CLN2) $0.7 \mathrm{U} / \mathrm{mg}$ of protein/h (corrected level: $54 \pm 18.2 \mathrm{U} / \mathrm{mg}$ of protein/h) was observed. Other metabolic, mitochondrial and mucopolysaccharidosis conditions have been excluded. The diagnosis was supplemented by numerous atrophic changes inside the brain, cerebellum, and widening of the central nervous system (CNS) ventricular spaces described in MRI.

Conclusions: An important element of the early diagnosis of CLN2 is the reduction of TPP1 activity with the characteristic eye fundus image that indicates the presence of buffalo maculopathy. An inseparable complement to the diagnosis of CLN2 is the fERG test indicating lesions of the rod-cone like system that underlie visual disturbances. KEY WORDS: neuronal ceroid lipofuscinosis, neuroimaging examinations, bull's eye maculopathy, retinal dystrophy, electrophysiology tests.

\section{INTRODUCTION}

Neurodegenerative diseases and neurological complications of inborn errors of metabolism (IEM) and storage disorders constitute about $38 \%$ of cases of genetically determined deaths [1]. In the group of neurodegenerative diseases there are neuronal ceroid lipofuscinoses (CLNs) - rare pediatric, chronic and progressive diseases associated with neurological and ophthalmic symptoms and leading to early death. Those diseases are most commonly inherited autosomally recessively, and are characterized by storage of lipofuscin in lysosomes [2]. There are currently over ten types of neuronal ceroid lipofuscinoses, but in the general population type 1 of the disease (CLN1, Santavouri-Haltii disease, HagbergSantavouri disease) is the most commonly observed, followed by type 2 (CLN2, Jansky-Bielschowsky disease), and type 3 (CLN3, Batten disease, Vogt-Spielmeyer disease, SpielmeyerSjögren disease) [3-6].

\section{CASE REPORT}

A 5-year-old male child was referred to the Pediatric Ophthalmology Ward with the diagnosis of CLN2 for a detailed ophthalmological examination. The child of the $2^{\text {nd }}$ pregnancy, born by natural labor at the age of $36 \mathrm{Hbd}$, with body weight of $2560 \mathrm{~g}$, Apgar score 8/9. Data from the medical history indicated normal development of the child until the age of 2 years. Over the age of 2 years, motor disorders of eyeballs were observed in form of exotropia, a discrete nystagmus and delayed development of active speech. Already at the age of 3 years neurological disorders were diagnosed, and EEG confirmed focal epilepsy with motor and vegetative seizures and consciousness disorders. Introduced anti-epileptic therapy proved unsuccessful. Aggressive behavior and regression in motor development manifested by difficulties with moving around were observed. Subsequently, arrest of motor development was noted with signs of ataxia of the trunk and limbs, 

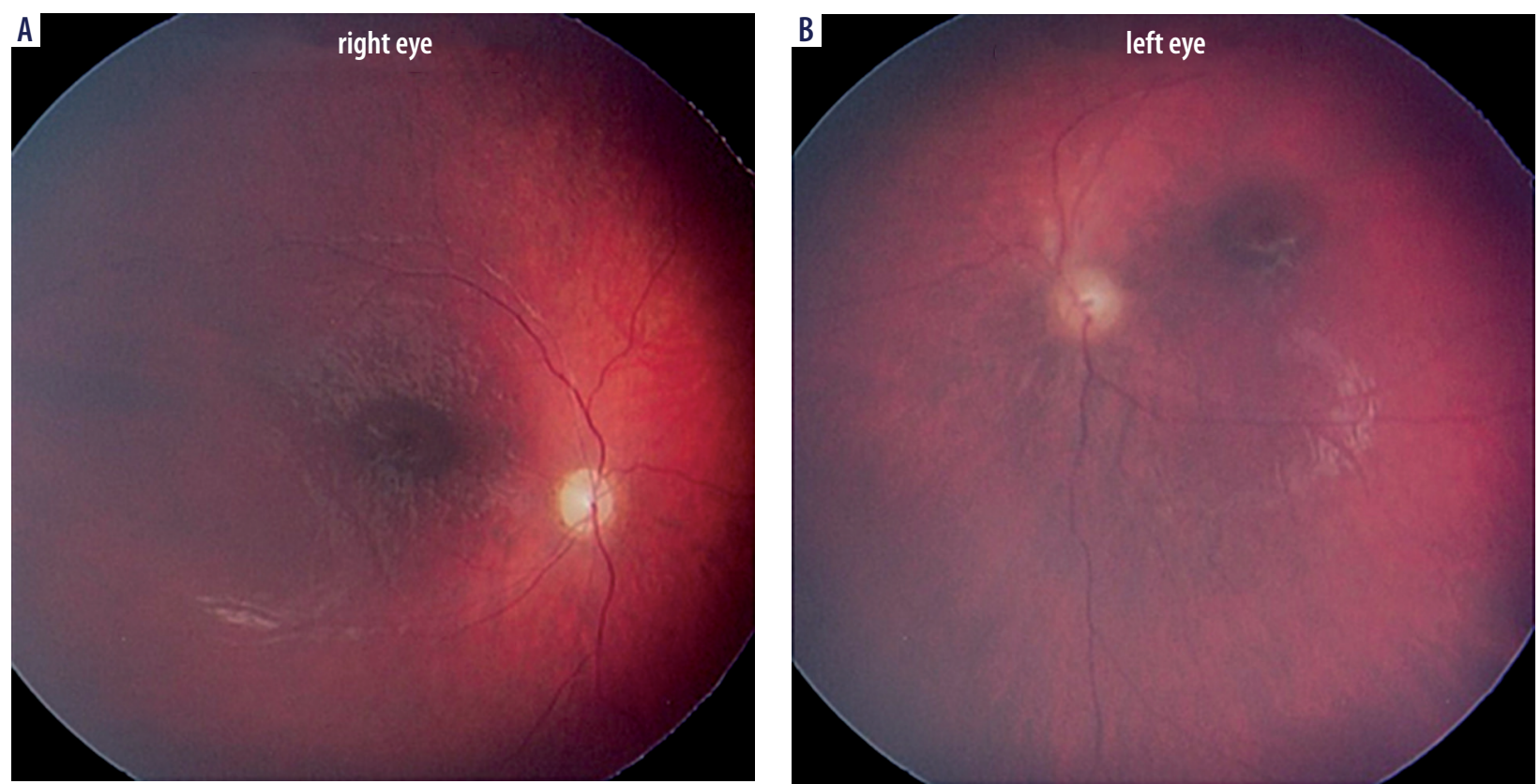

Figure 1. The eye fundus images in a child with CLN2. Bull's eye maculopathy, arteriolar narrowing and optic disc pallor are presented

contracture of ankles, feet positioned in plantar flexion, and reduced muscle tone. Circumference of the head was below the $3^{\text {rd }}$ percentile. Magnetic resonance imaging (MRI) of the cerebrum indicated signs of atrophy involving the cerebellum, with dilation of the ventricular system and damped signal from thalamuses in T2-weighted images. The EEG record was described as abnormal, with bilateral changes, often independent, localized in temporal-parietal-occipital areas, on many occasions occurring in synchronized manner, and generalized changes on the background of abnormal basic activity. US imaging of the abdomen and heart demonstrated no departures from the normal. ECG indicated the right axis of the heart and deep $S$ waves in V2-V4 leads. Extended diagnostics for neurodegenerative disorders excluded other metabolic, mitochondrial syndromes and mucopolysaccharidoses. Based on CNS lesions observed in the MRI and clinical symptoms, neurodegenerative disease was suspected. The diagnosis of CLN2 was confirmed by reduced activity of tripeptidyl-peptidase 1 - the lysosomal enzyme - in leukocytes in peripheral blood: 0.7 units/mg of protein/h (normal range: $54 \pm 18.2$ units/mg of protein/h).

The diagnosis was further supported by changes noted in ophthalmological examination: exotropia, discrete nystagmus with refraction error: hyperopia and astigmatism (RE - right eye: +2.5 Dsph/-2.5 Dcylax 27; LE - left eye: +2.5 Dsph/-3.25 Dcylax 136). Ret-Cam examination confirmed paleness of the optic disc, bullseye maculopathy, narrowing of arteries in the retina and irregular regrouping of pigment in the intermediate and far retinal circumference (Figure 1). fERG confirmed functional changes of retinal photoreceptors in form of cone rod dystrophy (Figure 2).

\section{DISCUSSION}

The clinical presentation of CLN2 is typical for progressive encephalopathy characterized by the onset of clinical symp- toms between 2 and 4 years of age. The first manifestation of the disease may be psychomotor anxiety, arrest and regression in psychomotor development, epileptic seizures of various morphology, mainly focal seizures, and speech development arrest. Psychomotor development is gradually inhibited, with progressive ataxia and constantly intensified myoclonic seizures. Children stop walking without support at the age of 4 to 6 years. Vision disorders gradually appear, leading to amblyopia and blindness around 6 years of age. A similar clinical course was observed in the discussed patient, in whom speech inhibition, decreased muscle tone and development of mental retardation were additionally observed. Patients with CLN2 usually die between the age of 6 and 12 years, as a result of rapid deterioration of all functions of the central nervous system, up to decortication and general cachexia [2]. The gold standard in laboratory diagnostics in the diagnosis of CLN2 is the reduced level of the tripeptidyl peptidase 1 (TPP1) enzyme (in leukocytes, fibroblasts and dried blood drops) or molecular identification of the mutation in each TPP1/CLN2 gene allele [7]. In the discussed case the TPP1 level was reduced down to $0.7 \mathrm{U} / \mathrm{mg}$ of protein/h (normal range: $54 \pm 18.2 \mathrm{U} / \mathrm{mg}$ of protein/h). The diagnosis is supplemented with results of neurophysiological tests, including EEG, fERG, as well as neuroimaging: axial computed tomography $(\mathrm{CT})$ of the brain, MRI $[2,8,9]$. The following data were obtained for the discussed patient: MRI indicating atrophic and neurodegenerative changes in the brain tissue, paroxysmal and generalized epileptic changes confirmed by EEG, and a decrease in the lysosomal activity of TPP1 demonstrated in laboratory investigations [7]. An ophthalmoscopic examination of the fundus showing characteristic changes in the retina and vessels at an early stage of the disease provides important complementary data for the final diagnosis of CLN2. In the discussed patient, Ret-Cam examination confirmed paleness of the optic disc, bullseye maculopathy, narrowing of arteries in the retina and 


\section{Right eye}

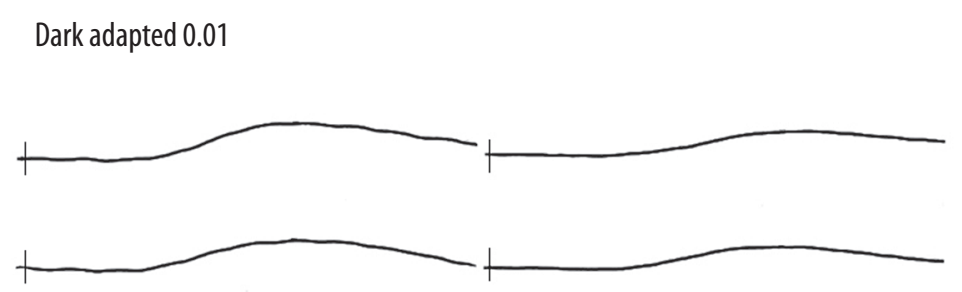

Dark adapted 3.0

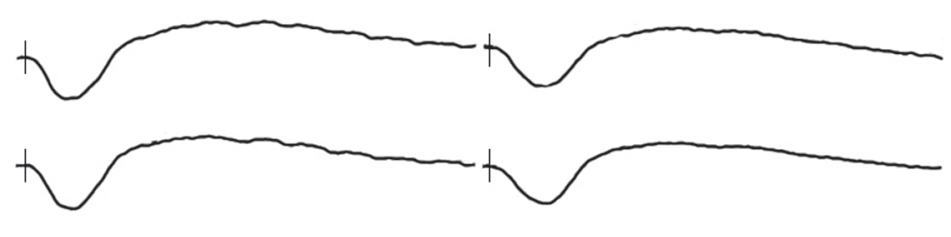

Dark adapted 3.0 - oscillatory potentials

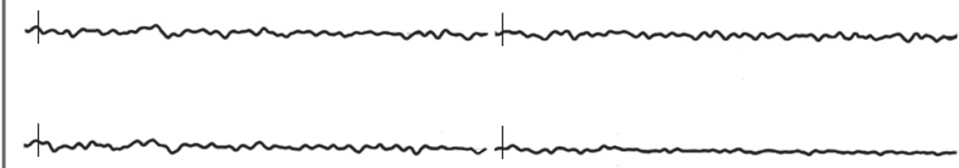

Light adapted 3.0

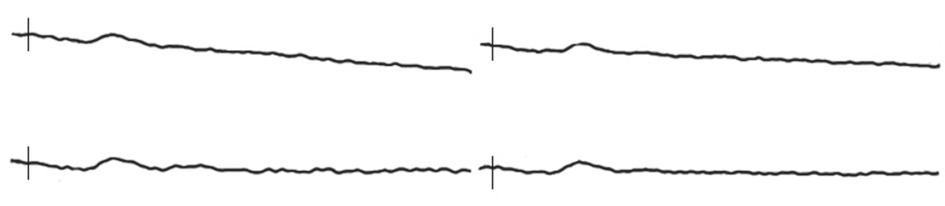

Light adapted flicker $30 \mathrm{~Hz}$

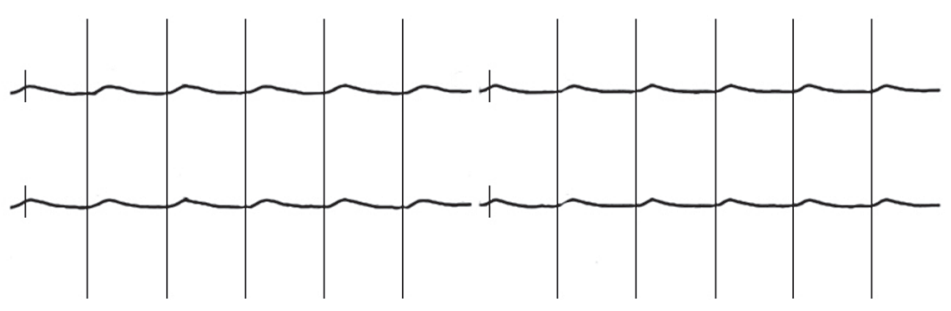

$100.0 \mu \mathrm{V}$

Control
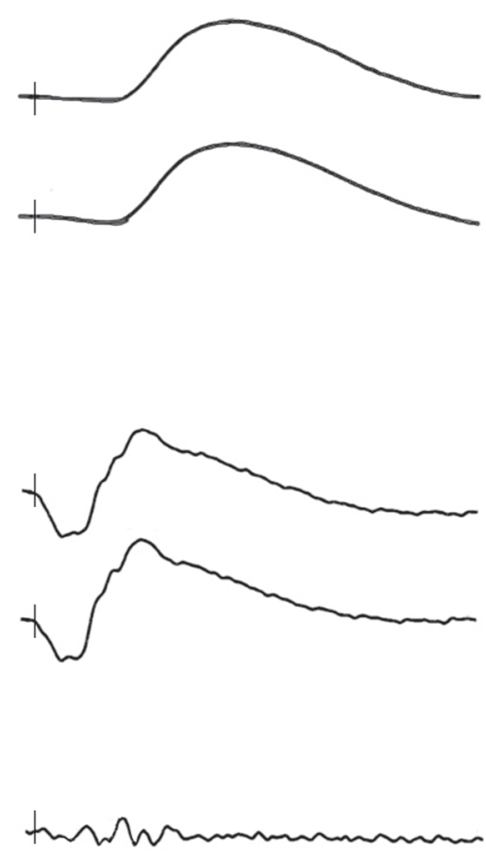

Thun
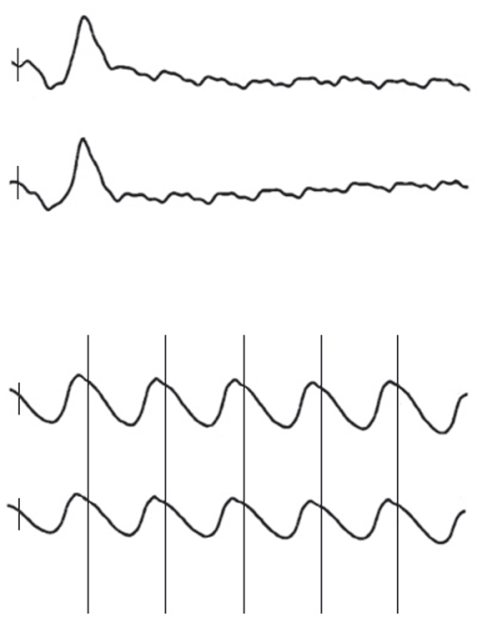

Figure 2. Flash ERG results in a CLN2 patient compared to a healthy person. Dominance of cone system damage - significant reduction of b wave in photopic response

irregular regrouping of pigment in the intermediate and far retinal circumference [8]. In electrophysiological fERG, damage to the retinal bioelectrical function was observed, to a greater extent in the cone photoreceptor system than in rods, indicating cone rod degeneration of the retina [2]. The fERG study is an important indicator for progressive metabolic changes in cone photoreceptors and retinal rods [10, 11]. In the early, asymptomatic phase of the disease, pathological changes are found in the form of a residual fERG record, which is completely extinguished (damage to cones and rods) after several years of the disease [2]. Differential diagnostics should take into account other genetically determined metabolic diseases, including Lennox-Gastaut epileptic syndrome, Tay-Sachs disease, sialidosis, Rett syndrome, metachromatic leukodystrophy, Krabbe disease, X-linked adrenoleukodystrophy, CGDs and other less common syndromes. 
Therapy in all forms of ceroid lipofuscinosis in children involves the use of drugs that interrupt seizures: valproic acid, topiramate, lamotrigine and other anti-epileptic drugs. Baclofen is also used in the treatment. The drug reduces muscle tone, and additionally has antimyoclonic and antidystonic effect. Despite the use of various antiepileptic drugs, results of antiepileptic treatment are unsatisfactory. Phenothiazine and midazolam derivatives are used in sleep disorders [2]. For the purpose of effective and targeted therapy, the patient was redirected from the Ophthalmology ward to the UKE center in Hamburg, offering treatment with Brineur - cerliponase alpha - a human TPP1 proenzyme that, due to its proteolytic activity, reduces accumulation of lysosomal material in nerve cells. The drug is administered directly into ventricles of the central nervous system, from a surgically implanted container [8]. Introduction of cerliponase alpha significantly slows progression of the disease in terms of motor functions and speech disorders in children with CLN2, as demonstrated in phase I/II trials [12-14].

Physiotherapy, orthoses and special orthopedic equipment are necessary for all patients in various stages of the disease; physiotherapy and ergotherapy in the form of various motor activities are particularly important. In the advanced stage of the disease, multidisciplinary medical assistance is necessary, with the help of a hospice [2].

In conclusion, it should be emphasized that the presence of vision disorders in the form of nystagmus, changes in the correct position of eyeballs and low visual acuity in a young child, that cannot be explained by a refractive error, is an indication for electrophysiological tests of VER and ERG. An abnormal result of these tests is an indication for extended diagnostics for neurodegenerative diseases. The disease is multidisciplinary, therefore a pediatric patient requires both neurological, pediatric and ophthalmological care.

\section{CONCLUSIONS}

Decrease in the TPP1 enzyme activity, progressive neurological and cognitive changes, epilepsy, confirmation of atrophic changes in various brain regions and characteristic bullseye changes in the macular region of the retina, with progressive decline in visual function are of key importance in making CLN2 diagnosis. fERG results indicating cone rod dystrophy additionally confirm visual impairment in CLN2.

\section{DISCLOSURE}

The authors declare no conflict of interest.

\section{References}

1. Rokicki D. Rozpoznanie wrodzonych chorób neurodegeneracyjnych na przykładzie choroby Niemanna-Picka typu C. Now Klik 2015; 23.

2. Kmieć T. Encefalopatie postępujące wieku dziecięcego uwarunkowane genetycznie. Wczesnodziecięca ceroidolipofuscynoza (CLN). Opieka Paliatywna nad Dziećmi 2007; 15: 115-120.

3. Yannuzzi LA. Atlas chorób siatkówki. Elsevier Urban \& Partner, Wrocław 2012.

4. Eaton SL, Proudfoot C, Lillico SG, et al. CRISPR/Cas9 mediated generation of an ovine model for infantile neuronal ceroid lipofuscinosis (CLN1 disease). Sci Rep 2019; 9: 9891.

5. Gardner E, Bailey M, Schulz A, et al. Mutation update: review of TPP1 gene variants associated with neuronal ceroid lipofuscinosis CLN2 disease. Hum Mutat 2019; 40: 1924-1938.

6. Dulz S, Wagenfeld L, Nickel M, et al. Novel morphological macular findings in juvenile CLN3 disease. Br J Ophthalmol 2016; 100: 824-828.

7. Gergont A, Kroczka S, Kaciński M. Przyczyny padaczki objawowej u dzieci w wieku 3-18 lat w latach 2006-2007. Przegl Lek 2008; 65: 751-757.

8. Fietz M, AlSayed M, Burke D, et al. Diagnosis of neuronal ceroid lipofuscinosis type 2 (CLN2 disease): Expert recommendations for early detection and laboratory diagnosis. Mol Genet Metab 2016; 119: 160-167.

9. Orlin A, Sondhi D, Witmer MT, et al. Spectrum of ocular manifestations in CLN2-associated batten (Jansky-Bielschowsky) disease correlate with advancing age and deteriorating neurological function. PLOS One 2013; 8: e73128.

10. Quagliato EMAB, Rocha DM, Sacai PY, et al. Retinal function in patients with the neuronal ceroid lipofuscinosis phenotype. Arq Bras Oftalmol 2017; 80: 215-219.

11. Weleber R. The dystrophic retina in multisystem disorders: The electroretinogram in neuronal ceroid lipofuscinoses. Eye 1998; 12: $580-590$.

12. CADTH Canadian Drug Expert Committee Recommendation: Cerliponase Alfa (Brineura — Biomarin Pharmaceutical [Canada] Inc.): Indication: For the treatment of neuronal ceroid lipofuscinosis type 2 (CLN2) disease, also known as tripeptidyl peptidase 1 (TPP1) deficiency. Ottawa (ON): Canadian.

13. Agency for Drugs and Technologies in Health 2019. Available from: http://www.ncbi.nlm.nih.gov/books/NBK543392/

14. Schulz A, Ajayi T, Specchio N, et al. Study of intraventricular cerliponase alfa for CLN2 disease. N Engl J Med 2018; 378: 1898-1907. 